\title{
UNA TAXONOMÍA DE LAS UNIVERSIDADES CHILENAS
}

\author{
Miguel Muñoz ${ }^{1}$, Christian Blanco²
}

\begin{abstract}
RESUMEN
Se propone una taxonomía de las universidades chilenas mediante análisis factorial, que resulta distinta a las tipologías de Brunner (2005, 2009), Parada (2010), Torres y Zenteno (2011) y Rosso y Reyes (2012), y supera las objeciones de Bernasconi (2006). Evita, además, distinciones binarias del tipo tradicional/privada, investigación/docencia, metropolitana/regional, católica/laica, etc. Tampoco establece rankings ni utiliza condiciones históricas, legales u otras alejadas de los modos de operación efectivos de las universidades. La siguiente taxonomía las agrupa según un pool de variables relevantes (investigación, tamaño, composición, acreditación, etc.) que da pie a cinco tipos de universidades: investigación, masivas, de acreditación, elitistas y no elitistas. Se espera aportar en la comprensión de las universidades y contribuir a las políticas públicas con herramientas conceptuales para abordar la diversidad universitaria.
\end{abstract}

Palabras clave: Clasificación, universidades, análisis factorial, Chile.

\section{TAXONOMY OF CHILEAN UNIVERSITIES}

\section{ABSTRACT}

We propose a taxonomy of Chilean universities through a factor analysis with different results from those produced by typologies of Brunner (2005, 2009), Parada (2010), Torres and Zenteno (2011) and Rosso and Reyes (2012), overcoming Bernasconi's objections (2006). It avoids binary distinctions like traditional/private, metropolitan/regional, catholic/secular, etc. It does not establish rankings nor use historical, legal or other distinctions not referred to the effective universities' operational characteristics. Universities are grouped according to a pool of relevant variables (research, size, composition, accreditation, etc.). Five types of universities are generated: Research, Massive, Accreditation, Elite and Non-Elite. This research intends to contribute to the understanding of universities in Chile and to public policies with conceptual tools for addressing the diversity of universities.

Keywords: Classifications, universities, higher education, Chile

1 Ingeniero Comercial y Licenciado en Ciencias Económicas y Administrativas, Pontificia Universidad Católica de Chile, Santiago, Chile. Contacto: mamunoz5@uc.cl

2 Licenciado en Filosofía, Licenciado en Sociología y Magíster en Análisis Sistémico Aplicado a la Sociedad, Universidad de Chile, Santiago, Chile. Contacto: chblancoj@gmail.com 


\section{UNA TAXONOMÍA DE LAS UNIVERSIDADES CHILENAS}

\section{Hacia una taxonomía de las universidades chilenas}

La creciente diversidad institucional de la educación superior a nivel global ha motivado una serie de esfuerzos de clasificación (Carnegie: Vega (2009)3); Stanley y Reynolds (1994), entre otras) y de rankings (como el Quacquarelli-Symonds-QS, Times Higher Education-THE, Academic Ranking of World Universities-ARWU, Ranking CHE y sus versiones ${ }^{4}$, entre otros), para entender de mejor manera el sistema de educación superior. La mayor parte de las clasificaciones y rankings dan cuenta más bien de la diversidad externa -diferencias entre instituciones- y no de la diversidad interna, relacionada con diferencias dentro de las instituciones (Birnbaum, 1983).

Los rankings buscan situar a las universidades en una «tabla de posiciones» de calidad, enfocada mayoritariamente hacia el desempeño en investigación (Van Vugh, J. Bartelse, J. Huisman M. y Van der Wende, 2010). Para algunos autores, como Van der Wende (2008), estos solo tendrían sentido entre grupos comparables de instituciones, por lo tanto, la clasificación sería un prerrequisito para un ranking, lo que implica una complementariedad entre ambas miradas. Por otra parte, Cheng y Cai Liu (2006) aplican una clasificación sobre los datos de rankings internacionales, realizando el camino inverso.

3 En el campo específico de las universidades, Vega (2009) clasifica taxonómicamente a las universidades privadas con fines de lucro de México en cuatro categorías o fases (emergentes, en transición, legitimadas y consolidadas), donde las instituciones pueden avanzar o retroceder en las fases. Esta clasificación no explicita el método utilizado para la generación de la taxonomía y las categorías y subcategorías perfiladas son de difícil comprensión.

4 Los esfuerzos de los proyectos U-Map y U-Multirank se encuentran en una posición intermedia entre clasificación y ranking, ya que asumen la existencia de diversos perfiles institucionales. U-Multirank, en particular, ranquea por desempeño según las categorías del U-Map. 
En general, el sentido de los esfuerzos de clasificación a nivel mundial dice relación con la necesidad de entender mejor la diversidad de misiones y perfiles institucionales de la educación superior, haciéndolos más transparentes (Van Vugh, 2005; 2007; 2009). Las clasificaciones pueden tener fines investigativos, para la comprensión de la estructura del sistema; administrativos, para la asignación de recursos o bien, políticos, para la justificación de decisiones (McCormick, 2012). Aunque es más habitual en los rankings que en las clasificaciones, a veces se incluye también el criterio de desempeño (Cheol Shin, 2009).

En Chile hay experiencias tanto de clasificaciones (Brunner, 2005; 2009; Torres y Zenteno, 2011) como de rankings de universidades (revista Qué Pasa, revista América Economía, etc.). Respecto de las clasificaciones, en general estos esfuerzos son más bien ejercicios tipológicos (ideográficos) basados en clasificaciones conceptuales más que taxonómicos (nomotéticos), es decir, derivados del análisis de datos empíricos (Bailey, 1994) respecto de las universidades, donde ambas aproximaciones envuelven una teoría de la diferenciación implícita o explícita (Brint, 2006).

No es posible determinar a priori ni definitivamente si procede utilizar una tipología o una taxonomía en determinado caso. En las tipologías no es difícil entender cómo son categorizadas las entidades, lo que permite una cierta flexibilidad en la selección de atributos o datos de clasificación, pero su justificación empírica puede ser limitada o no existente. En cambio, las taxonomías implican una mayor dificultad de entendimiento para casos específicos o limítrofes y exigen la disponibilidad y utilización de los mismos datos para todas las entidades, a su favor, se puede afirmar que se encuentran empíricamente mejor justificadas (McCormick, 2012).

La presente investigación analiza críticamente los intentos previos de clasificación chilenos y los inscribe dentro de un modelo evolutivo de las clasificaciones de universidades, al tiempo que propone una nueva clasificación de carácter taxonómico para las universidades chilenas, esfuerzo pionero en esta materia. 
Debido a que los conceptos utilizados para realizar las clasificaciones mencionadas provienen en gran medida de un arrastre histórico de distinciones respecto de las universidades, estas no hacen referencia directa a las condiciones de operación efectiva o misión que las caracterizan. El uso de elementos como la estructura jurídica, el régimen de propiedad, la localización geográfica, la confesión religiosa católica o su condición de laica, entre otras distinciones similares tienden a ocultar los modos efectivos de funcionamiento, los productos, servicios y los enfoques que se relacionan directamente con el desarrollo de su misión institucional (Brint, 2006). Las condiciones de operación, aunque estén en alguna medida incluidas en tales esfuerzos clasificatorios chilenos previos, siguen bajo una mirada conceptual y no empírica o estadística. En este sentido, y dada la experiencia internacional (Brint, 2006; McCormick, 2012; Ziegele, 2012; Van Vught, 2005; 2007; 2009; Van Vught et al 2010, entre otros), parece necesario avanzar en clasificar universidades en función de la develación de estructuras subyacentes mediante análisis de datos, más que desde las distinciones tradicionales del lenguaje cotidiano, habituales en el debate institucional y en las políticas de asignación de recursos actuales.

La siguiente taxonomía de las universidades chilenas es replicable en otros países (sujeta a disponibilidad de datos), usa una innovadora metodología de análisis de factores y obtiene resultados de clasificación distintos a Brunner (2005; 2009), Parada (2010), Torres y Zenteno (2011) y Rosso y Reyes (2012), superando las objeciones de Bernasconi (2006) a este tipo de ejercicios tipológicos.

Proponemos ir más allá de las distinciones binarias habituales de universidades de tipo tradicional/privada, estatal/privada, investigación/docencia, selectiva/no selectiva, metropolitana/regional, católica/laica, entre otras. Asimismo, tampoco tiene el sentido de un ranking, que lejos de asumir la complejidad, simplificaría la diversidad en una escala unidimensional continua, para establecer comparaciones verticales, tratando de identificar mejores y peores universidades (Ziegele, 2012). La siguiente propuesta multidimensional no utiliza condiciones históricas, legales y otras distintas a la misión de las universidades, sino que las agrupa según sus características 
distintivas dentro de un pool de variables relevantes identificadas en la literatura (investigación, tamaño, composición, acreditación, etc.). La clasificación emerge del análisis y validación de los datos de universidades chilenas, sin suponer conceptos o categorías previas al procesamiento de los mismos. Esto permite a la taxonomía favorecer comparaciones horizontales entre grupos, identificando similitudes y diferencias (Brint, 2012; Ziegele, 2012).

El resultado del estudio es una clasificación de las universidades chilenas en cinco grupos, según las condiciones de operación que las caracterizan y que las diferencian de otros grupos. Estos son: 1) universidades de investigación, 2) universidades masivas, 3) universidades de acreditación, 4) universidades elitistas y 5) universidades no elitistas. Internamente, en cada categoría se esbozan nuevas subclasificaciones solo a modo de propuesta para la discusión. Esta clasificación no tiene un sentido de distinción de niveles de calidad ni de establecimiento de un ranking, sino meramente de la indicación de lo que caracteriza a cada grupo según sus condiciones de funcionamiento, denotando la diversidad externa del sistema universitario chileno.

La investigación espera ser un aporte en la comprensión de las universidades en las condiciones actuales de desarrollo de la educación superior en Chile, cuya heterogeneidad difícilmente puede reducirse a las distinciones tradicionalmente utilizadas. Aunque es un trabajo estrictamente académico, espera contribuir también a las políticas públicas con nuevos insumos de clasificación, en la medida en que estas se ven enfrentadas a una creciente diversidad en el sistema.

El hecho de contar con una clasificación taxonómica de universidades no significa un desconocimiento de la importancia de las tipologías que se han construido previamente ni de la relevancia que tienen las distinciones de carácter histórico. De hecho, se considera que este esfuerzo es heredero de los intentos de clasificación previos y que una reflexión respecto de la realidad de las universidades en Chile, y en cualquier otro país, debe considerar ambas formas de clasificación. De hecho, la distinción público/privado (Brunner et al, 2011), cuya relevancia permanece para el debate sobre las 
universidades chilenas -y también latinoamericanas- se pone en perspectiva a la luz de los resultados de la presente investigación. Las clasificaciones basadas en análisis de datos empíricos, como el análisis de factores y clustering, cuestionan a aquellas provenientes del lenguaje natural y de los conceptos sedimentados de uso social cotidiano. Del mismo modo que constituye un error taxonómico considerar que los murciélagos son «ratones con alas» -es decir, roedores y no quirópteros- no parece conveniente continuar utilizando acríticamente las distinciones tradicionales para clasificar universidades en la actualidad.

\section{Evolución de las clasificaciones de universidades}

El sistema de educación superior chileno ha evolucionado de manera vertiginosa, experimentando grandes cambios en los últimos treinta años, lo cual ha resultado en un panorama universitario de creciente complejidad y alta heterogeneidad. En este escenario existe el riesgo de que los conceptos, distinciones y clasificaciones que se utilizan para dar cuenta de esta diversidad hayan perdido su potencia explicativa, convirtiéndose en anacrónicos respecto de la realidad que se quiere representar. Por ello, los esfuerzos para clasificar a las universidades deben ser permanentes y, en lo posible, depender más del análisis de los datos y menos de las distinciones de uso tradicional.

Actualmente, asistimos a la sedimentación de una semántica (Luhmann, 2007; Dockendorff, 2006) de la clasificación de las universidades sobre la base de criterios institucionales, de propiedad, de afinidad religiosa y criterios geográficos, entre otros. Los discursos tanto de los actores del mundo político-legislativo, del sector académico, de los líderes estudiantiles, de los administradores de las políticas públicas y de la ciudadanía operan fundamentalmente distinguiendo a las universidades bajo el par tradicionales/privadas. Sobre esta clasificación culturalmente consolidada se desarrollan los otros criterios, que incluso las clasificaciones más sistemáticas y formalizadas hasta el momento tienden a reproducir. Sin embargo, hoy las condiciones de operación de las universidades en Chile permiten deducir una clasificación independiente de la distinción tradicional/ privada o cualquiera de sus extensiones. 
No es ajeno a la teoría social el hecho de que ciertas distinciones semánticas tengan intereses de grupos específicos que pugnan por perpetuar la hegemonía de algunos conceptos, o bien, que buscan generar otra distinción que los favorezca. De hecho, «siempre, en toda sociedad, hay conflictos entre poderes simbólicos, que apuntan a imponer una visión de divisiones legítimas» (Bourdieu, 1989, p. 22). El debate acerca de la clasificación de universidades no es la excepción, ya que también está integrado en la dinámica en la que se distribuye el poder político e ideológico (Brint, 2006). Por la naturaleza del ejercicio tipológico, es más vulnerable a ser permeado por las distinciones tradicionales vinculadas con conflictos por el poder simbólico. Por el contrario, el análisis taxonómico basado en técnicas cuantitativas reduce en gran medida este riesgo, permitiendo la emergencia de estructuras -en un principio- sin el insumo conceptual del investigador.

Los esfuerzos de clasificación de universidades, por tanto, pueden inscribirse en un esquema evolutivo según el criterio de distinción utilizado para construir las categorías. Un análisis general permite identificar tres grandes momentos: 1) etapa histórica, 2) etapa tipológica y 3) etapa taxonómica. El hecho de distinguir tres etapas cronológicamente sucesivas no implica que las distinciones se hayan dejado de utilizar o hayan perdido total validez, sino que cada momento ofrece una complejización acumulativa de las posibilidades de comprensión del sistema de universidades en Chile.

En la etapa histórica de clasificación -que no fue producto de un debate académico sino que de una condición histórica y políticapredomina una distinción general del sistema de universidades, sedimentado en los discursos sociales y que articula las concepciones actuales del panorama universitario en Chile, basada en el par universidad tradicional/privada.

A partir de la nueva institucionalidad de la educación superior chilena impuesta en 1981, se estableció la distinción entre las universidades creadas antes de la ley -como las estatales o aquellas privadas con un cierto carácter público-y aquellas creadas después de la ley de 1981: las nuevas universidades privadas. Sobre la distinción 
principal se establecieron otras distinciones como aquella respecto del régimen de propiedad: estatal/privada. Lo mismo ocurrió con la agrupación legal y organizacional de las instituciones, definiéndose algunas como parte del Consejo de Rectores de las Universidades Chilenas y otras no (CRUCH/no CRUCH), lo cual determinó a su vez políticas de financiamiento y gobierno diferenciadas para ambos tipos de instituciones, las que se mantienen hasta el día de hoy. Igualmente operan sobre esta clasificación otros pares que tomaron relevancia con posterioridad, que también dicen relación con aspectos estructurales, legales u otros distintos de sus condiciones de operación. Tal es el caso de la distinción según ubicación geográfica, metropolitana/regional, o según afinidad religiosa, católica/no católica o laica.

Las distinciones de dicho momento de la evolución en la clasificación de universidades, basadas en condiciones históricoinstitucionales -adscritas, no influenciables, fijas- comenzaron a ser insuficientes para identificar a los grupos de estas. Algunas instituciones privadas comenzaban a elevar su selectividad y otras a desarrollar algún nivel de investigación, de modo que la denominación tradicional/privada y otras similares tendieron a quedar obsoletas para denotar tales diferencias. Es decir, ninguna de estas distinciones, ni la combinación de las mismas, parecían necesarias ni suficientes para generar una clasificación de universidades con alta capacidad de discriminación y con resultados intuitivos y de fácil comprensión. Aunque estas distinciones pueden indicar algunas de las características de las universidades, no agrupan unívocamente a las instituciones y no dan cuenta de sus condiciones de operación o su misión. Ante esta necesidad, comenzaron a aparecer los esfuerzos de clasificación más académicos, basados en nuevas tipologías que tienden a superar las distinciones binarias de este primer periodo.

La segunda etapa es la tipológica. Esta constituye un momento intermedio entre la etapa histórica y la taxonómica. A diferencia de la anterior, este periodo emerge como una reacción de algunos investigadores que comienzan a seleccionar variables relativas a las condiciones de funcionamiento y desarrollan grandes avances al pasar de distinciones binarias a distinciones en varias dimensiones, que incluyen el análisis de datos empíricos. 
El primer esfuerzo en esta línea fue el de Brunner (2005), que en su libro Guiar el mercado ofrece una tipología de universidades en ocho categorías, usando una combinación de nociones binarias propias de la etapa anterior y de datos sobre matrícula, publicaciones, programas y otras variables asociadas. El autor reconoce que las clasificaciones empleadas en ese momento -tanto en el lenguaje oficial como en el mercado (como con los rankings, por ejemplo)no pueden dar cuenta de la realidad del sistema. La clasificación de Brunner distingue nítidamente entre universidades públicas y privadas, y emplea el nivel de selectividad 5 de las universidades como patrón principal de clasificación, bajo el supuesto -ampliamente documentado en tal informe- de que estas operan en un mercado jerárquicamente estructurado. Además, busca determinar las funciones sociales que juegan las distintas categorías de universidades, atendiendo tanto a su naturaleza selectiva como a los elementos subyacentes de selectividad social. Al mismo tiempo, procura incluir un rango amplio de variables de clasificación a fin de poder reflejar la diferenciación y heterogeneidad de las instituciones universitarias, lo que constituye su mayor aporte. Sin embargo, combina insumos tradicionales conceptuales (estatuto legal, misión declarada, prestigio y regionalidad) con nuevos insumos empíricos (tamaño de matrícula, cobertura de áreas profesionales, selectividad e investigación). Al respecto, Bernasconi (2006) señala la existencia de ambigüedades, confusiones con el criterio de selectividad, deficiencias en la nomenclatura, no inclusión de la variable «clase social» y otras críticas a la clasificación, concluyendo que le parece más apropiada «una clasificación que ponga el acento en las funciones y resultados de la universidad, porque [...] estos aspectos tienen más que ver con la identidad y la misión real de una universidad» (Bernasconi, 2006, p. 84). Ante estas y otras observaciones, Brunner (2009) repite el ejercicio tipológico proponiendo una clasificación de siete categorías que agregan la variable de la composición socioeconómica del alumnado, manteniendo distinciones binarias de la etapa histórica. Sin embargo, el autor sigue utilizando un análisis conceptual sobre

5 Una medida tradicional de la selectividad es el porcentaje de alumnos que está dentro de los 27.500 mejores puntajes en la PSU, los cuales reciben AFI. En Brunner (2009) y en este estudio se asume esta forma de operacionalización. 
los datos -y no un análisis estadístico de los mismos- como base de su clasificación.

También inscrito en la etapa tipológica, Parada (2010) propone una categorización tridimensional basada en las distinciones de tipo de propiedad, organización jurídica, y productos y servicios que ofrece una universidad ${ }^{6}$. El ejercicio concluye en 40 subcategorías, agrupables luego en tres grandes tipos de universidades: completas (públicas puras, privadas puras y semipúblicas), docentes (docentes públicas, docentes privadas) y de investigación. El autor termina ofreciendo una categorización que resuena en gran medida con las distinciones históricas asociadas a la forma de entrega de recursos por parte del Estado (tradicional/privada), combinándola con la distinción binaria de mayor actualidad: investigación/docencia.

Torres y Zenteno (2011) realizan un nuevo intento de clasificación, esta vez considerando a la selectividad y a la investigación como los factores de distinción principales. Los autores recuperan las variables utilizadas por Brunner $(2005 ; 2009)$ y establecen una tipología de siete grupos sin subcategorías. La categorización da una relevancia primordial al par investigación/docencia y al nivel de selectividad. Sin embargo, presenta los mismos problemas que las clasificaciones anteriores, como por ejemplo, poca claridad en los nombres, injustificados criterios de clasificación y heterogeneidad interna de los grupos de universidades. Además, no se consideran otras variables relevantes como la expansión (medida según proporción de matrícula nueva) y características socioeconómicas (como el establecimiento de origen), que ya habían sido identificadas como relevantes por Brunner (2009).

Uno de los trabajos de punta en el tema de la clasificación de universidades en Chile es el de Rosso y Reyes (2012), en la medida en que comienza no solo a analizar conceptualmente los perfiles empíricos de las instituciones, sino que incluye criterios estadísticos y cálculos matemáticos para construir su clasificación, pero manteniendo la

6 Aunque el autor declara un propósito de taxonomía, al no realizar un análisis de datos cuantitativos termina proponiendo más bien una tipología, según la distinción de Bailey (1994). 
distinción investigación/docencia como criterio binario principal, comparando las universidades de manera vertical más que horizontal. Se hace una segmentación a priori y luego se clasifica a las instituciones según tal esquema. Aunque constituye un intento incipiente de taxonomía, la investigación de Rosso y Reyes, al intentar establecer cual universidad es mejor en cada agrupación, se asemeja más bien a un «ranking categorizado». De hecho, para cada grupo se toma como tipo ideal aquel que lidera la categoría. Finalmente, termina con el establecimiento del concepto de pares académicos para hacer estudios entre tipos de universidades comparables. Así como Parada (2010), estos autores orientan su clasificación para el uso en políticas públicas y entrega de financiamiento, aunque otros han advertido acerca de los riesgos de este uso de las clasificaciones (Ziegele, 2012).

En varios de los ejercicios tipológicos de clasificación revisados subyace la idea de que hay universidades mejores o más completas, acercándose a la idea de un ranking o una tabla de comparación vertical, ya que las universidades pueden inscribirse en un continuo único que sugiere niveles de calidad o deseabilidad crecientes.

En síntesis, la evolución en las clasificaciones de las instituciones ha pasado por tres momentos. En la etapa histórica, periodo comprendido entre la reforma de 1981 hasta la primera mitad de la década de 2000, la clasificación depende exclusivamente del estatus institucional de las universidades, siendo la distinción tradicional/privada la más importante. Luego, en la etapa de clasificación tipológica -el segundo momento- esta se complejiza, ya que se dispone de mayor cantidad de información existe también mayor relevancia y heterogeneidad externa entre las universidades privadas y del $\mathrm{CRUCH}$, pero se mantiene el peso de los elementos legales, valóricos y otros. En general, la importancia de las variables cuantitativas como matrícula y selectividad se combinan con distinciones geográficas, institucionales y religiosas. Con la presente investigación se abre una nueva etapa caracterizada por esfuerzos de clasificación taxonómica. En parte prefigurado por la propuesta de Rosso y Reyes (2012), en el desarrollo de este tercer momento los factores adscritos a las universidades pierden relevancia frente a otros elementos distintos de los criterios institucionales, dando lugar 
a clasificaciones de carácter estadístico. Este esfuerzo pretende dar un impulso en la consolidación de esta nueva fase en la evolución de las clasificaciones de universidades en Chile.

\section{Construcción de una taxonomía con análisis factorial}

El análisis de factores ha servido como herramienta de clasificación en diferentes campos, incluyendo la educación superior. Lagrosen S., Seyyed-Hashemi, R. y Leitner, M. (2004) examinan las dimensiones de calidad en la misma y las comparan con las dimensiones generales de calidad de servicio, aplicando el análisis de factores sobre resultados de encuestas. Villela y Hu (1991) diseñan un modelo conceptual de la retención de estudiantes no tradicionales y examinan el modelo de forma empírica, usando dicha metodología para entender los factores subyacentes al fenómeno. El análisis de factores también ha sido utilizado para clasificar entidades en la biología (Hirzel, 2002), la economía (Aguirre y Céspedes, 2004), la psicología (Briggs y Cheek, 1986), el comportamiento organizacional (Banks y Greggs, 1965), la antropología (Driver y Schuessler, 1957) y la ciencia política (Rummel, 1971), entre otros campos.

No se ha encontrado evidencia dentro de la bibliografía revisada de clasificaciones de universidades con análisis factorial. La mayoría de ellas son tipológicas (conceptuales), sin embargo, existen esfuerzos de clasificaciones taxonómicas que usan fundamentalmente cluster analysis, entre los que se cuentan: Huberty C., Jordan, E. y Brandt W. (2005), Brint (2006), Cheol Shin (2009), Bonaccorsi y Daraio (2009), entre otros.

La presente investigación es de tipo cuantitativa, no experimental, transversal y de carácter descriptivo y su objetivo es construir una taxonomía de universidades. El análisis de datos se realizó por medio de la técnica estadística multivariada reductiva denominada «análisis factorial con el método de factor principal de carácter exploratorio». La investigación trabajó sobre datos secundarios de diversas fuentes, algunos entregados por las propias universidades y otros generados por diversos organismos públicos 
vinculados con la educación superior chilena. Los datos pasaron por diversos procesos de validación en los organismos proveedores.

La base de datos incluye información de 55 universidades de un total de 59 vigentes al año 2010. Esta diferencia se origina por el hecho de no contar con información para ciertas variables para la totalidad de las universidades ${ }^{7}$. Dicho tamaño se ajusta a la regla general del análisis factorial de contar con, al menos, cuatro o cinco veces más observaciones -en este caso, universidades- que el número total de variables. La base de datos consiste en 15 variables de uso extendido en investigaciones de caracterización y clasificación de universidades (Brunner, 2005; 2009; Torres y Zenteno, 2011; Rosso y Reyes, 2012). Las variables, según fuente de origen, son las siguientes:

Tabla 1: Variables de la base de datos según fuente de información

\begin{tabular}{|c|c|}
\hline Sedes & CNED 2010 \\
\hline Programas de pregrado & CNED 2010 \\
\hline Programas de magíster & CNED 2010 \\
\hline Programas de doctorado & CNED 2010 \\
\hline Acreditación institucional & CNA 2010 \\
\hline Acreditación por área & CNA 2010 \\
\hline Matrícula total & SIES 2010 \\
\hline Matrícula de primer año (nueva) & SIES 2010 \\
\hline Titulados de pregrado & SIES 2010 \\
\hline Proyectos Fondecyt & CNED 2010 \\
\hline Publicaciones & TR-ISI 2010 \\
\hline Selectividad & SIES-MECESUP 2010 \\
\hline Matrícula nueva de origen municipal & SIES 2010 \\
\hline Matrícula nueva de origen particular subvencionado & SIES 2010 \\
\hline Matrícula nueva de origen particular pagado & SIES 2010 \\
\hline \multicolumn{2}{|c|}{$\begin{array}{l}\text { Notas: } \\
\text { CNA: Comisión Nacional de Acreditación } \\
\text { CNED: Consejo Nacional de Educación } \\
\text { SIES: Servicio de Información de Educación Superior } \\
\text { MECESUP: Programa de Mejoramiento de la Calidad y Equidad de la Educación Superior } \\
\text { TR-ISI: Thompson Reuters - Web of Knowledge (ex Institute for Scientific Information, ISI) }\end{array}$} \\
\hline
\end{tabular}

Fuente: Elaboración propia

Para ser ingresadas al modelo, todas las variables se consideraron en términos totales, sin aplicarse estandarizaciones.

7 No se dispone de información para: Universidad Mayor, Universidad Gabriela Mistral, Universidad La República y Universidad Los Leones. 
Para el perfil estadístico se utilizan promedios en algunas variables que tienen sentido para su presentación.

El análisis de factorial exploratorio permite determinar la estructura subyacente en la matriz de datos respecto de las universidades chilenas (Gorsuch, 1983). Esta técnica se caracteriza porque no se conoce de antemano el número de factores y es en su aplicación empírica donde se determina dicho número. Esto significa que la presente investigación renunció a predeterminar el número de factores a priori -ya que esto se asocia a un análisis de tipo confirmatorio- permitiendo la emergencia autónoma de factores sobre los cuales se construye la clasificación.

La taxonomía, a diferencia de la tipología, usualmente se desarrolla con uso de computador y apoyo de un software especializado. Para la generación de los factores se utilizó el programa estadístico STATA 11.0, específicamente por medio del comando factor con la opción del factor principal pf (principal factor method). Este es un método de obtención de la matriz factorial en el que los factores explican la máxima varianza y son incorrelacionados ${ }^{8}$. Siempre proporciona una solución, por lo cual se asume la probabilidad de generar estimadores sesgados en la matriz de cargas factoriales, si existen variables con comunalidades bajas.

$\mathrm{Al}$ aplicar la regla de scree plot-donde se recomienda cortar en aquel factor donde los valores propios tienen un punto de inflexión' el análisis factorial resultó en cinco factores:

8 Otro método utilizado para la obtención de la matriz de carga factorial es el de máxima verosimilitud, destacando la posibilidad de este para formular test de hipótesis sobre la estructura factorial.

9 Notar que el factor 5 tiene un valor cercano a uno, valor de corte para la regla de Kaiser. El método de Kaiser y scree plot son los dos más utilizados, aunque es importante señalar que existen otras técnicas para la estimación del número de factores como el método de Bartlett y el análisis paralelo. 
Tabla 2: Valores propios por factor y proporción de la varianza explicada

\begin{tabular}{|l|c|c|c|c|}
\hline Factor & Valor propio & Diferencia & Proporción & Prop. acumulada \\
\hline Factor 1 & 6,58 & 3,29 & 0,49 & 0,49 \\
\hline Factor 2 & 3,29 & 1,73 & 0,24 & 0,73 \\
\hline Factor 3 & 1,56 & 0,55 & 0,12 & 0,84 \\
\hline Factor 4 & 1,01 & 0,09 & 0,07 & 0,92 \\
\hline Factor 5 & 0,92 & 0,73 & 0,07 & 0,99 \\
\hline Factor 6 & 0,19 & 0,11 & 0,01 & 1,00 \\
\hline Factor 7 & 0,08 & 0,01 & 0,01 & 1,01 \\
\hline Factor 8 & 0,07 & 0,05 & 0,01 & 1,01 \\
\hline Factor 9 & 0,02 & 0,02 & 0,00 & 1,01 \\
\hline Factor 10 & 0 & 0 & - & 1,01 \\
\hline Factor 11 & 0 & 0,01 & - & 1,01 \\
\hline Factor 12 & $-0,01$ & 0,03 & $-0,00$ & 1,01 \\
\hline Factor 13 & $-0,04$ & 0 & $-0,00$ & 1,01 \\
\hline Factor 14 & $-0,04$ & 0,03 & $-0,00$ & 1,01 \\
\hline Factor 15 & $-0,07$ & - & $-0,01$ & 1,00 \\
\hline
\end{tabular}

Fuente: Elaboración propia.

Al establecer el corte en el quinto factor por medio del comando factors (5) se verifica el cumplimiento del criterio de porcentaje de la varianza. Este indica el número mínimo de factores necesario para explicar un porcentaje acumulado satisfactorio de la varianza del modelo, que debe encontrarse, al menos, sobre un $75 \%$ o un $80 \%$. En este caso, al quinto factor, la varianza acumulada que explica el modelo es del 98,4\%.

En general, la matriz de cargas factoriales obtenidas no define factores interpretables, para ello se realiza una rotación con el fin de obtener factores cuya matriz de cargas factoriales sea más comprensible. Por medio del procedimiento de rotación se trata de conseguir que unas saturaciones sean altas a costa de que otras bajen, para así destacar la influencia de los factores comunes sobre las variables observables (Cuadras, 2012). El comando que se utiliza es rotate, que permite obtener una solución final de agrupación de variables a cada factor. Adicionalmente se ocupó el método de rotación varimax ${ }^{10}$, que produce una rotación ortogonal, donde los ejes se giran de tal forma que quede preservada la correlación entre factores. Este método minimiza el número de variables con cargas

10 Otro método de rotación de factores es la rotación oblicua. 
altas en un factor, mejorando así la capacidad de interpretación de los factores.

Al reflexionar teóricamente sobre las variables asociadas a un determinado factor, inmediatamente se encuentran rasgos comunes que permiten su identificación, que ya puede recibir una denominación adecuada.

Por último, el comando predict calcula una puntuación de regresión o regression scoring para asignar un puntaje a cada una de las instituciones por factor, permitiendo asociar cada universidad al factor en que obtenga mayor puntaje, es decir, asociar la universidad al que mejor la caracterice. Algunas instituciones (menos del 10\% del total) no pudieron ser asignadas simplemente analizando los puntajes de regresión, dada la similitud entre el puntaje para cada uno de los factores (cuatro casos «limítrofes»). Estas instituciones de borde se asignaron al grupo más similar según un análisis comparativo del perfil estadístico de las universidades, dando lugar a resultados intuitivos y que cumplen con el criterio de interpretabilidad. Esto es una situación habitual en el análisis factorial cuando hay factores ortogonales, donde se puede dar lugar a puntuaciones correlacionadas, siendo una de las limitaciones de estos modelos estadísticos. Otra alternativa hubiese sido eliminar los casos de borde, lo que implicaría una pérdida de información que afectaría negativamente la comprensibilidad general de la taxonomía, por lo que se decidió incluirlos en el grupo más afín.

Otro aspecto relevante es que el método de rotación de factores permite aproximarse a la solución obtenida por el principio de estructura simple (Thurstone, 1935), que señala que la matriz de cargas factoriales debe reunir las siguientes características que se verifican en el modelo (ver Tabla 3): (1) cada factor debe tener unos pocos pesos altos y los otros próximos a cero, criterio que se cumple; (2) cada variable no debe estar saturada más que en un factor, criterio que también se cumple, salvo para la variable selectividad; (3) no deben existir factores con la misma distribución, vale decir, dos factores distintos deben presentar distribuciones diferentes de cargas altas y bajas, criterio que también se cumple. 


\section{Taxonomía de las universidades chilenas}

La taxonomía resultante del análisis factorial sugiere la existencia de cinco factores, dados los valores propios de cada uno (Eigenvalue). Según las variables que agrupan a cada factor, es posible asignar denominaciones teóricas que permitan su interpretación en la forma de una clasificación de universidades. A continuación se presentan cada uno de los factores ordenados de manera decreciente según la proporción que explica cada uno en la varianza del modelo:

- Factor 1: se caracteriza por la alta importancia de las publicaciones científicas y proyectos Fondecyt, seguido de los programas de postgrado, especialmente doctorados y también magíster. Cabe destacar la carga relativamente alta que tiene la variable selectividad.

- Factor 2: este factor se encuentra definido principalmente por el tamaño de la matrícula nueva y de la matrícula total, denotando masividad, seguido del gran número de programas de pregrado. El número de sedes también es una variable con carga relativamente alta para este factor.

- Factor 3: este factor se caracteriza por tener altas cargas en la acreditación institucional y la acreditación por área. Adicionalmente, poseen una alta carga en selectividad, pero menor a la de las variables señaladas anteriormente.

- Factor 4: este factor se define principalmente por dos variables que son las que tienen mayor carga, relacionadas con la proveniencia de colegios particulares pagados y la alta selectividad.

- Factor 5: este factor se caracteriza por la alta importancia que tiene el establecimiento de origen, en este caso, municipal. Le sigue la variable sedes. Otro aspecto que lo define es la carga negativa que tiene en la variable selectividad.

Las asociaciones de las variables en cada factor se pueden apreciar en la siguiente tabla, que ha sido ordenada según la importancia del factor en el modelo y según las variables que se le asocian a cada uno: 
Tabla 3: Carga de factores por variables ordenada por factor

\begin{tabular}{|l|c|c|c|c|c|}
\hline Variables & Factor 1 & Factor 2 & Factor 3 & Factor 4 & Factor 5 \\
\hline Publicaciones & 0,96 & 0,14 & 0,15 & 0,10 & $-0,03$ \\
\hline Fondecyt & 0,95 & 0,13 & 0,17 & 0,12 & $-0,03$ \\
\hline Magíster & 0,74 & 0,28 & 0,36 & 0,21 & $-0,18$ \\
\hline Doctorado & 0,90 & 0,16 & 0,29 & 0,07 & $-0,03$ \\
\hline Sedes & $-0,18$ & 0,56 & $-0,37$ & 0,16 & 0,46 \\
\hline Programas pregrado & $-0,11$ & 0,84 & $-0,20$ & $-0,05$ & 0,25 \\
\hline Matrícula total & 0,39 & 0,85 & 0,29 & 0,00 & $-0,03$ \\
\hline Matrícula nueva & 0,26 & 0,92 & 0,15 & 0,01 & $-0,05$ \\
\hline Titulados & 0,38 & 0,70 & 0,20 & 0,02 & 0,11 \\
\hline Acred. instituciones & 0,32 & 0,10 & 0,86 & 0,16 & $-0,12$ \\
\hline Acreditación áreas & 0,32 & 0,12 & 0,85 & 0,10 & 0,01 \\
\hline Selectividad & 0,56 & 0,03 & 0,55 & 0,41 & $-0,20$ \\
\hline Part. pagado & 0,13 & $-0,04$ & 0,11 & 0,74 & $-0,65$ \\
\hline Municipal & $-0,03$ & 0,08 & $-0,04$ & $-0,13$ & 0,98 \\
\hline Part. subvencionado & $-0,17$ & $-0,01$ & $-0,13$ & $-0,97$ & 0,05 \\
\hline
\end{tabular}

Fuente: Elaboración propia.

Al comparar estos resultados con otros esfuerzos de clasificación chilenos, llama la atención que no exista la preponderancia de una o algunas variables en todos los factores (agrupaciones) o en la mayor parte de estos, como sí ocurre en los otros esquemas revisados. Desde esta perspectiva, el modelo propuesto estaría considerando la heterogeneidad del sistema de un modo consistente. El sistema de universidades en Chile es heterogéneo, pero no al nivel reportado en la literatura, en la que se proponen clasificaciones entre siete y ocho categorías, utilizando variables similares (Brunner, 2005; 2009; Torres y Zenteno; 2011).

Para aquellos que clasifican unidimensionalmente (por ejemplo, por nivel de investigación, como Rosso y Reyes, 2012) o tridimensionalmente (propiedad, organización y productos y servicios; Parada, 2010) se obtiene un número similar de grupos. Por el contrario, la taxonomía propuesta en esta investigación, con un gran número de variables, genera un número moderado de agrupaciones, cumpliendo también con el criterio de parsimonia.

A partir de las características de los factores, de los puntajes resultantes del regression scoring por universidad y del análisis de los perfiles estadísticos para los casos de borde, es posible asignar los 
conceptos correspondientes a los factores para denotar las estructuras subyacentes develadas por el análisis factorial, lo que equivale a construir la taxonomía:

Tabla 4: Denominación taxonómica de factores subyacentes

\begin{tabular}{|l|l|}
\hline Factor 1 & Universidades de investigación \\
\hline Factor 2 & Universidades masivas \\
\hline Factor 3 & Universidades de acreditación \\
\hline Factor 4 & Universidades elitistas \\
\hline Factor 5 & Universidades no elitistas \\
\hline
\end{tabular}

Fuente: Elaboración propia.

Al clasificar las universidades en cada uno de los factores es posible adicionalmente esbozar una subcategorización interna de los factores dadas las variables principales de cada uno, solo como ejercicio para la discusión. El ejercicio taxonómico puede extenderse sobre la base de distinciones de niveles dentro de la primera variable de clasificación por factor, utilizando análisis cuantitativo, pero no estadístico multivariable; en este caso se adoptó como metodología la división en tramos según los valores de la variable más distintiva. En el caso de las universidades no elitistas, esto debió realizarse atendiendo a los valores de la segunda variable más característica (selectividad) buscando mayor interpretabilidad. Debido a que en el grupo de universidades de investigación solo tres quedan clasificadas, no tiene mucho sentido hacer tramos, lo que sí puede realizarse con los factores 2 y 5 .

1. Universidades de investigación: conformado por la Universidad de Chile, la Pontificia Universidad Católica de Chile y la Universidad de Concepción, ordenadas en orden decreciente por número de publicaciones. La categoría se caracteriza por tener un alto número de programas de postgrado, con una participación del 26\% del total de los programas de magíster y del 46\% en programas de doctorado. Adicionalmente, son las que tienen un mayor número de proyectos Fondecyt y de publicaciones, con una participación del 57\% y $69 \%$ del total, respectivamente. Tienen los índices de selectividad más altos del sistema (47,8\%) y sus alumnos provienen principalmente de establecimientos particulares subvencionados. 
2. Universidades masivas: compuesto por nueve instituciones que se caracterizan por tener una gran participación en la matrícula nueva y total del sistema, alcanzando un 32\% y 33\% del total, respectivamente. Son instituciones eminentemente de pregrado con un gran número de sedes -llegando a un promedio de 9,4 sedes por institución-y cuyos alumnos son mayoritariamente de establecimientos particulares subvencionados, superando el 55\% de participación. Podemos clasificar internamente a este grupo en tres subcategorías de acuerdo con su matrícula nueva en orden decreciente:

a) Masividad alta (mayor a 8.000 matriculados nuevos). Grupo compuesto por Universidad Nacional Andrés Bello y la Universidad Tecnológica de Chile, INACAP.

b) Masividad media (entre 5.000 y 8.000 matriculados nuevos). Grupo compuesto por la Universidad Santo Tomás, la Universidad de las Américas, la Universidad San Sebastián, la Universidad del Mar y la Universidad Autónoma de Chile.

c) Masividad moderada (menos de 5.000 matriculados nuevos). Compuesto por la Universidad Central y la Universidad Arturo Prat. Ambas instituciones tienen una matrícula nueva cercana a los 3.900 alumnos.

3. Universidades de acreditación: este grupo se compone por 20 instituciones caracterizadas por contar en gran medida con la acreditación institucional y otras áreas específicas acreditadas, teniendo por lo general muchos años de funcionamiento dentro del sistema. También tienen una alta actividad de postgrado, participando en un 38\% de los programas de magíster y un $43 \%$ de los de doctorado respecto del total. Son el segundo grupo con mayor nivel de proyectos FONDECYT y publicaciones, muy por sobre el resto de los demás grupos. Su índice de selectividad llega al 20,5\%. La proveniencia de sus alumnos es principalmente de establecimientos particulares subvencionados, alcanzando aproximadamente un 45\%. Al generar subcategorías durante los años de acreditación institucional, se producen los siguientes grupos internos:

a) Acreditación alta (igual a seis años). Subgrupo compuesto por la Pontificia Universidad Católica de Valparaíso, la Universidad 
Católica del Norte, la Universidad de Santiago de Chile, la Universidad Austral de Chile y la Universidad Técnica Federico Santa María, todas universidades pertenecientes al CRUCH.

b) Acreditación media (entre cinco y cuatro años). Categoría integrada por la Universidad de La Frontera, la Universidad Alberto Hurtado, la Universidad Católica del Maule, la Universidad Católica de Temuco, la Universidad de Tarapacá, la Universidad de Talca, la Universidad de Antofagasta, la Universidad Católica de la Santísima Concepción, la Universidad de La Serena, la Universidad Metropolitana de Ciencias de la Educación y la Universidad Católica Silva Henríquez. En este subgrupo, si bien es heterogéneo, tienen alta participación las universidades católicas y aquellas formadas a partir de la escisión de las sedes regionales de la Universidad de Chile y de la Universidad Técnica del Estado.

c) Acreditación moderada (entre dos y tres años). Compuesta por la Universidad de Playa Ancha de Ciencias de la Educación, la Universidad de Valparaíso y la Universidad Tecnológica Metropolitana. Todas son instituciones pertenecientes al CRUCH.

4. Universidades elitistas: si bien este grupo tiene un bajo número promedio de sedes -es decir, se encuentran bastante concentradas- se caracterizan principalmente por tener un alto índice de selectividad, el cual llega aproximadamente a un 29\% (el segundo más alto) y una alta proveniencia de alumnos de establecimientos particulares pagados, cercana al 46\%. Por otra parte, el número de programas de pregrado es bastante similar a los de postgrado y la acreditación institucional bordea los seis años en promedio. Este grupo lo componen solo universidades privadas y es posible generar subcategorías dentro de él de acuerdo con la participación que tengan los alumnos provenientes de establecimientos particulares pagados (ordenadas de manera decreciente):

a) Altamente elitistas (participación superior a un 70\% de establecimientos particulares pagados). Lo componen la Universidad de los Andes, Universidad Adolfo Ibáñez y Universidad del Desarrollo. 
b) Elitistas (participación entre un 70\% y 45\% de establecimientos particulares pagados), grupo compuesto por la Universidad Finis Terrae y la Universidad Diego Portales.

5. Universidades no elitistas: este grupo se caracteriza eminentemente por un gran número de programas de pregrado y varias sedes en promedio. Tienen el más bajo nivel de acreditación institucional y solo por áreas. En promedio, también tienen el nivel más bajo de matrícula total, no superando los 5.000 alumnos por institución, es decir, son universidades pequeñas. Se caracterizan principalmente por tener un bajo índice de selectividad (menor a $2 \%$ en promedio) y la proveniencia de sus alumnos es principalmente de establecimientos municipales. Podemos subcategorizar el grupo según selectividad (ordenados de manera creciente). Las subcategorías son las siguientes:

a) No selectivas (menos del 1\%). Se encuentra compuesta por la Universidad Miguel de Cervantes, la Universidad de las Ciencias de la Informática, la Universidad Bolivariana, la Universidad Internacional SEK, la Universidad de Los Lagos, la Universidad Iberoamericana de Ciencias y Tecnología, la Universidad Bernardo O'Higgins y la Universidad de Artes y Ciencias Sociales, en su mayoría universidades privadas.

b) Baja selectividad (entre un 3\% y 1\%). Este grupo se compone de la Universidad Pedro de Valdivia, la Universidad Adventista de Chile, la Universidad de Viña del Mar, la Universidad Chileno Británica de Cultura, la Universidad Academia de Humanismo Cristiano y la Universidad de Artes, Ciencias y Comunicación, todas universidades privadas.

c) Moderada selectividad (entre un 6\% y 3\%). Se compone de la Universidad de Magallanes, la Universidad del Pacífico y la Universidad de Atacama. Destaca la participación de dos universidades del $\mathrm{CRUCH}$.

El perfil estadístico por institución incluye las 15 variables del modelo, más dos variables adicionales -el número de instituciones y el promedio de matrícula total-, lo cual permite entender y comparar los grupos de instituciones. 


\section{Hacia una nueva etapa en la clasificación de universidades}

La taxonomía abre una nueva etapa en la clasificación de universidades en Chile, más allá de la fase histórica de distinciones binarias y de la fase tipológica donde se introducen los datos empíricos. Los resultados de esta investigación indican que el sistema de universidades es diverso, pero no en la medida de lo propuesto por investigaciones previas, que derivan hasta siete u ocho categorías distintas con variables similares. Las universidades chilenas se pueden clasificar en cinco categorías no ordenables jerárquicamente ni describibles unívocamente bajo ningún criterio binario. Respecto de la principal distinción estatal/privada, por ejemplo, se observa que hay universidades estatales clasificadas en cuatro de las cinco categorías. Estas dan cuenta de distintas estructuras de funcionamiento o misiones de las universidades chilenas, mostrando las similitudes y diferencias entre ellas.

Ahora bien, ¿por qué es importante evaluar la diversidad externa en educación superior? ¿Qué significa contar con una gran diversidad de universidades? Birnbaum (1983) y la adaptación de Van Vught (2007) a estos postulados, presentan una visión a favor de la diversidad externa. Muchos de estos argumentos aparecen como altamente relevantes en el contexto del diseño de políticas en educación superior.

En primer lugar, un sistema más diversificado supone tener más capacidad de ofrecer acceso a educación superior a los estudiantes de diferentes niveles educativos y con una variedad de historias de vida y de logros académicos. El argumento es que en un sistema diversificado con desempeño variable, a cada estudiante se le ofrece la oportunidad de participar y competir con estudiantes de antecedentes similares (Van Vught, 2007). El contraargumento puede ser que un sistema diversificado tiende a la segregación estudiantil de distintos niveles socioeconómicos, académicos y culturales, lo que finalmente impediría el desarrollo de diversidad interna. La existencia de un grupo de universidades no elitistas y otro elitista en la taxonomía propuesta abre la discusión sobre lo conveniente o no de este tipo de diferenciación para el sistema chileno. 
Un segundo argumento es que la diversidad favorece la movilidad social, permite correcciones de las elecciones, entrega oportunidades adicionales y amplía los horizontes educacionales (Van Vught, 2007). Por otra parte, esta diversidad puede conducir a niveles de calidad diferentes, sin garantías de mejorar las oportunidades.

Tercero, la diversidad supone satisfacer las necesidades del mercado del trabajo mejor que los sistemas homogéneos, promoviendo el desarrollo económico (Van Vught, 2007). Sin embargo, la diversidad no implica que los egresados se inserten exitosamente en el mercado laboral. En la taxonomía, la existencia de un importante número de universidades masivas puede implicar un riesgo de desbalance entre oferta y demanda por capital humano, lo que abre el debate acerca de la «cesantía ilustrada».

El cuarto argumento recogido por Birnbaum es que la diversidad sirve a las necesidades políticas de grupos de interés, en la medida en que un sistema diverso asegura la representación de intereses de diferentes grupos sociales, para su propia identidad y legitimación (Van Vught, 2007). Esto involucraría que las universidades funcionarían también como centros de reproducción ideológica no completamente dedicados al uso y producción del conocimiento. Esto abre el debate respecto de las universidades elitistas de la taxonomía, ya que en ellas puede observarse un cierto predominio de una visión ideológico-religiosa particular y de un grupo socioeconómico más privilegiado, vinculado con el empresariado. Por otra parte, no hay garantías de que en el sistema universitario la totalidad de los intereses sociales queden representados.

Un quinto argumento es que la diversidad permite la combinación de una educación superior de élite con una de masas (Van Vught, 2007). Los sistemas masivos tienden a ser más diversificados que los sistemas de élite. Esta visión de coexistencia de sistemas se asocia más a sistemas de mercado, lo que se refleja claramente en la taxonomía (investigación, masivas, de acreditación, élite y no élite), pero puede entrar en conflicto con las expectativas de algunos movimientos estudiantiles y sociales en Chile, que demandan 
una educación superior gratuita y de calidad para todos, entendida como derecho y no como bien de consumo.

Una sexta razón por la que la diversidad es un importante objetivo es que se asume que esta aumenta el nivel de especialización y, por tanto, de efectividad de las universidades (Van Vught, 2007). Sin embargo, la taxonomía de las universidades chilenas muestra especialización (entendida como menor variedad de programas de pregrado) solo en las universidades elitistas. Por el contrario, para las universidades masivas la diversidad se expresa en un gran número de programas de pregrado y no en especialización. La eficiencia, en este caso, se entendería de manera genérica como el desarrollo de economías de escala o liderazgo en costos, y no como la especialización o diferenciación (Porter, 2007). Esta discusión evidencia la necesidad de avanzar en estudios de diversidad interna de las universidades.

Finalmente, se supone que la diversidad ofrece oportunidades para experimentar con la innovación (Van Vught, 2007). En un sistema diversificado, las universidades tienen la opción de evaluar la viabilidad de las innovaciones creadas por otras instituciones, sin necesariamente tener que implementarlas ellas mismas. Las condiciones de benchmark no las puede resolver completamente la taxonomía -porque se requiere de análisis de la diversidad interna-, pero sin duda permite, al menos, una mayor comprensión de la diversidad externa.

Esta nueva perspectiva de clasificación posibilita el que sean los datos los que revelen los tipos de universidades y no los conceptos o conocimientos previos que se tengan de ellos, poniendo en cuestión la vigencia de los mismos para interpretar la creciente complejidad del panorama universitario nacional. Sin embargo, este ejercicio es heredero de los momentos anteriores en la evolución de la clasificación de universidades. Por una parte, el modelo utiliza variables relevadas en la literatura teórica, lo que termina favoreciendo la consistencia y la interpretabilidad de los resultados. Además, la clasificación propuesta reduce el número de grupos, avanzando en parsimonia y comprensibilidad. 
Con estos resultados, nuevas investigaciones y programas de política pública cuentan con herramientas de carácter científico para el análisis del sistema universitario en Chile, lo que se estima un aporte tanto en términos teóricos como prácticos, en la medida en que esta clasificación puede ser utilizada como nueva variable de cruce, como criterio de análisis institucional, etc. Ziegele (2012) reconoce algunos usos adecuados adicionales desde las políticas públicas, como la dirección de la inversión según perfiles o focos de interés, la adaptación de las mediciones de desempeño y calidad a los distintos perfiles, así como el hecho de contar con una base de conocimiento para acordar objetivos con las universidades. Sin embargo, no se recomienda usar las clasificaciones para implementar nuevos tipos legales, para vincular indicadores directamente con el financiamiento, para controlar el acceso a la ayuda estudiantil, para medir calidad o para reemplazar la discreción de los policy-makers por automatismos estrictos orientados según las categorías de la clasificación.

Futuras investigaciones podrán incorporar datos acumulativos y comparativos para una serie de años, profundizando en la dinámica del proceso de diferenciación. Al respecto, McCormick (2012) sugiere como «caminos hacia la excelencia» el fortalecimiento de la investigación y/o el incremento de la selectividad, que podrían ser observados por medio de movimientos de universidades entre las categorías de la taxonomía. Para identificar estos procesos de diferenciación -y verificar la sensibilidad a los cambios, la consistencia de las categorías y la robustez del modelo en el tiempo- la taxonomía debería reproducirse utilizando series de años para periodos más largos. El presente estudio operó solo sobre la base de datos de 2010, año relativamente estable en la institucionalidad universitaria, previo a la crisis de la Universidad del Mar y a las irregularidades detectadas en el sistema de acreditación. Si bien de un año a otro los perfiles estadísticos tienden a ser relativamente estables, estas coyunturas $-\mathrm{y}$ la acumulación de transformaciones en el mediano plazo- eventualmente podrían afectar ciertas variables en algunas universidades, lo que hace relevante extender esta investigación en el futuro, para observar elementos de cambio y de permanencia en el sistema universitario. 
El hecho de contar ahora con una taxonomía de las universidades no significa desconsiderar la complejidad al interior de los grupos de universidades, en la medida en que otros elementos cruzan diversos tipos de instituciones, como por ejemplo, el caso de la selectividad. Asimismo, aunque el modelo pone en perspectiva la validez de las clasificaciones previas, no las omite ni las descarta, sino que ofrece una posibilidad de interpretación de las distinciones tipológicas tradicionales al interior de las categorías de la taxonomía.

Entendiendo que lo que se percibe como real, es real en sus consecuencias (Merton, 1995), las distinciones tradicionales siguen plenamente vigentes en la discusión académica y en la acción en políticas públicas. Se expresan en los esquemas de financiamiento, en los medios de comunicación y en los discursos institucionales. En este sentido, uno de los valores de esta taxonomía es permitir la observación de tales distinciones, para ponerlas en perspectiva y, quizás, llegar a superarlas.

\section{Referencias}

Aguirre, A. y L. Céspedes, (2004) Uso de análisis factorial dinámico para proyecciones macroeconómicas. Revista Economía Chilena, 7(3), pp. $35-46$

Bailey, K., (1994) Typologies and taxonomies. An introduction to classification techniques. Papers $\mathrm{n}^{\circ}$ 102. Disponible en http://www.sagepub.com/ booksProdDesc .nav? contribId=502366\&prodId=Book4445\#tabvie $\mathrm{w}=$ title

Banks, A. y D. Greggs, (1965) Grouping political systems: Q - factor analysis of a cross-polity Survey. American Behavorial Scientist, 9. Pp. 3-6

Bernasconi, A., (2006) La difícil tarea de clasificar universidades. Calidad en la Educación (25), pp. 81-96

Birnbaum, R., (1983) Maintaning Diversity in Higher Education. San Francisco: Jossey-Bass.

Bonaccorsi, A. y C. Daraio, (2009) Characterizing the european university system: a preliminary classification using census microdata. Science and Public Policy, 36 (10), pp. 763-775.

Bourdieu, P., (1989) Social space and symbolic power. Sociological Theory, 7.(1), pp. 14-25. 
Briggs, S. y J. Cheek, (1986) The role of factor analysis in the development and evaluation of personality scales. Journal of Personality. 54 (1) pp. 106-148.

Brint, S., (2012) Reference sets, identities \& aspirations in U.S. higher education. International Conference Ministry of Education, Chile.

Brint, S.; Riddle, M. \& R. Hanneman, (2006) Reference sets, identities, and aspirations in a complex organizational field: The case of American four-year colleges and universities. Sociology of Education, 79, pp. 126-140.

Brunner, J. J.; Elacqua, G.; Tillett, A.; Bonnefoy, J.; González, S.; Pacheco, P. y F. Salazar, (2005) Guiar el mercado. Informe sobre la educación superior en Chile. Viña del Mar (Chile), UAI.

Brunner, J. J., (2009) Tipología y características de las universidades chilenas. Documento para comentarios. Disponible en http://200.6.99.248/ bru487cl/files/2009/02/post_116.html

Brunner, J. J. y C. Peña, (eds.) (2011) El conflicto de las universidades: Entre lo público y lo privado. Ediciones Universidad Diego Portales, Santiago de Chile, 2011, pp. 9-61.

Cheng, Y. y N. Cai Liu, (2006) A first approach to the classification of the top 500 world universities by their disciplinary characteristics using scientometrics. Scientometrics, 68 (1), pp. 135-150.

Cheol Shin, J., (2009) Classifying higher education institutions in Korea: a performance-based approach. Higher Education, 57, p p. 247-266.

Cuadras, C., (2012) Nuevos métodos de análisis multivariante. CMC: Barcelona.

Dockendorff, C., (2006) Evolución de la cultura: la deriva semántica del cambio estructural. Persona y Sociedad, 20 (1), pp. 45-73

Driver, H. y K. Schuessler, (1957) Factor analysis of ethnographical data. American Anthropologist, 59 (4), pp. 655-663.

Gorsuch, R., (1983) Factor analysis. (2a ed.). L. Erlbaum Associates: New Jersey.

Hirzel, A.; Hausser, J.; Chessel, D. y N. Perrin, (2002) Ecological-Niche factor analysis: how to compute habitat-suitability maps without absence data? Ecology, 83, pp. 2027-2036

Huberty, C.; Jordan, E. y W. Brandt, (2005) Cluster analysis in higher education. Higher Education: Handbook of Theory and Research, 20, pp. 437-457.

Logrosen, S.; Seyyed-Hashemi, R. y M. Leitner, (2004) Examination of 
the dimensions of quality in higher education. Quality Assurance in Education, 12 (2), pp. 61-69.

Luhmann, N., (2007) La sociedad de la sociedad. México: Herder.

McCormick, A., (2012) Classifying higher education institutions: lessons from the Carnegie classification. Center for Postsecondary Research. Indiana University, School of Education Bloomington. International Conference Ministry of Education, Chile.

Merton, R., (1995) The Thomas theorem and the Matthew effect. Social Forces, 74(2), pp. 379-424.

Parada, J., (2010 Universidades públicas y privadas: un enfoque tridimensional. Disponible en http://www.cepchile.cl/1_4745/doc/universidades_ publicas_y_privadas_un_enfoque_tridimensional.html

Porter, M., (2007) Competitive strategy. Executive Education: New York.

Rosso, P. P. y C. Reyes, (2012) Una nueva clasificación de las universidades chilenas. En proceso de publicación.

Rummel, J., (1971) Dimensions of error in cross-national Data. In a Handbook of Method in Cultural Anthropology, R. Naroll and R. Cohen (Eds). Garden City, New York: Natural History Press, 1971, pp. 946-961.

Stanley, G. y P. Reynolds, (1994) Similarity grouping of Australian universities. Higher Education, 27(3), pp. 359-366.

Thurstone, L., (1935) The vectors of mind: multiple-factor analysis for the isolation of primary traits. Chicago, IL, US: University of Chicago Press.

Torres, R. y E. Zenteno, (2011) El sistema de educación superior. Una mirada a las instituciones y sus características. En Foro Aequalis "Nueva Geografía de la Educación Superior y de los Estudiantes. Una cartografía del sistema chileno, su actual alumnado y sus principales tendencias", Jimenez, M y Lagos. F (Eds.). Ediciones Universidad San Sebastián, pp. 13-72.

Van der Wende, M., (2008) Rankings and classifications in higher education: a european perspective, en J. C. Smart (ed.) Higher Education: Handbook of Theory and Research. Dordrecht: Springer, pp. 49-71.

Van Vught, F.; J. Bartelse; J. Huisman y M. Van der Wende, (2005) Institutional profiles, towards a typology of higher education institutions in Europe. IAU Horizons, 13 (2), pp. 9-11

Van Vught, F., (Ed.) (2009) Mapping the higher education landscape: towards a European classification of higher education. Springer: London.

Van Vught, F.A.; Kaiser, F.; File, J.M.; Gaethgens, C.; Peter, R. y D.F. Westerheijden, (2010) U-Map. The European classification of Higher 
Education Institutions. CHEPS, Enschede. Disponible en: http://www.umap.org/U-MAP_report.pdf

Vega, G., (2009) Poniendo orden a las instituciones particulares de educación superior en México: una taxonomía aplicada a su complejidad y diversidad. Revista de Educación Superior, 38 (150) pp. 37-60.

Villela, E. y M. Hu, (1991) A factor analysis of variables affecting the retention decision of nontraditional college students. NASPA Journal, 28, (4), pp.334-344.

Ziegele, F., (2012) Classification of higher education institutions: the European case. Center for Higher Education (CHE). International Conference Ministry of Education, Chile. 

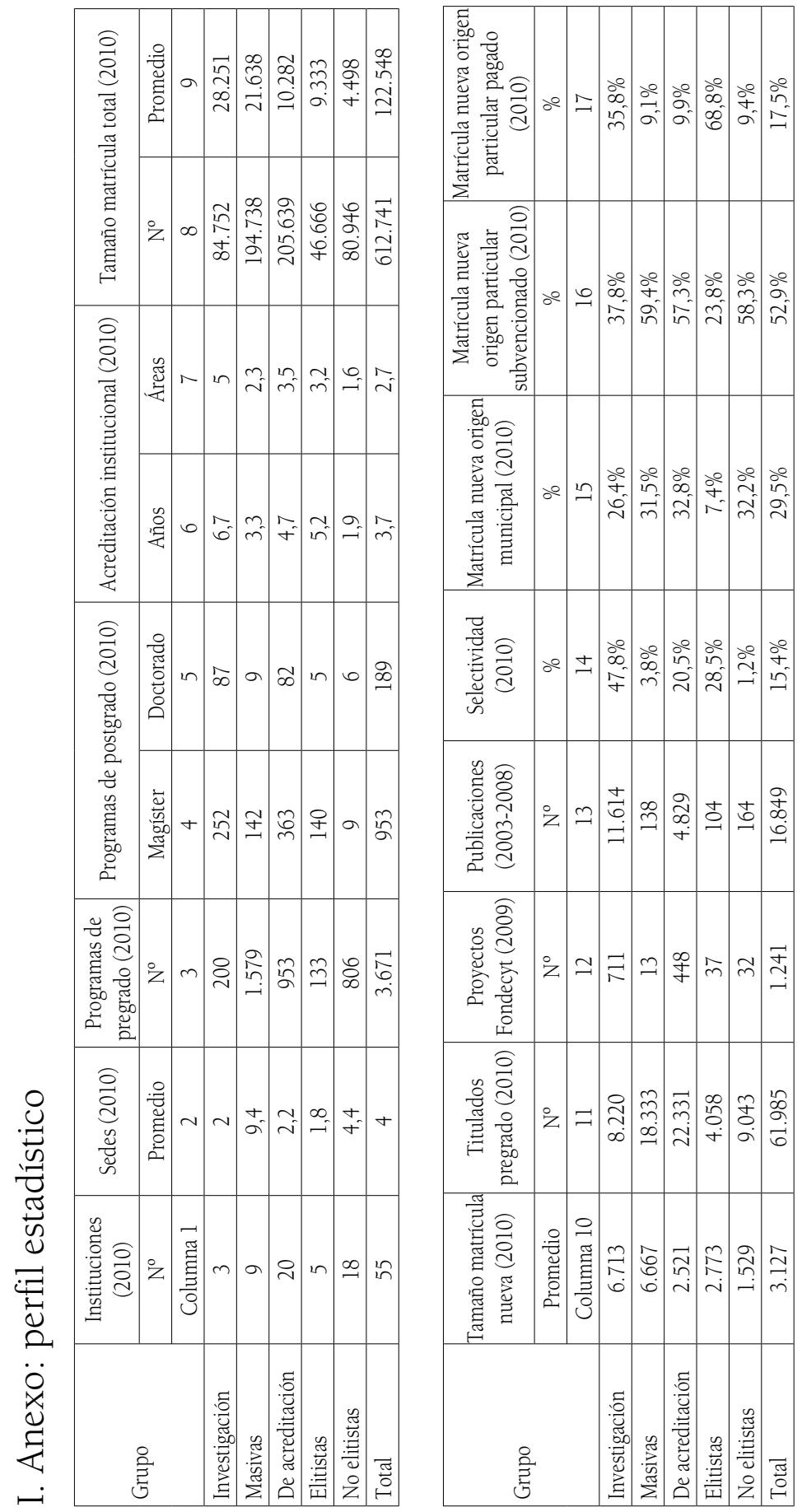

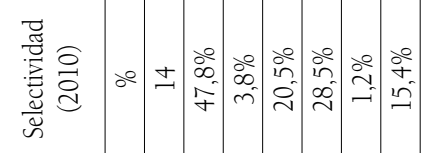

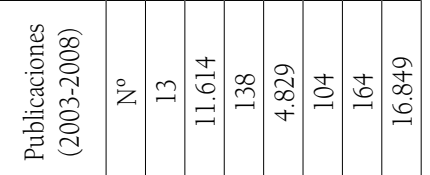

\begin{tabular}{|c|c|c|c|c|c|c|c|}
\hline 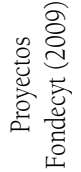 & iz & $\beth$ & $\exists$ & $\approx$ & 番 & $\hat{m}$ & $\approx$ \\
\hline
\end{tabular}

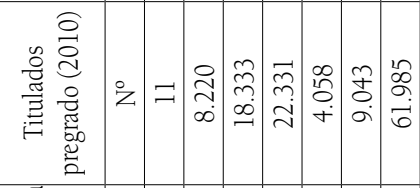

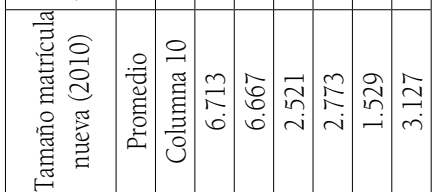

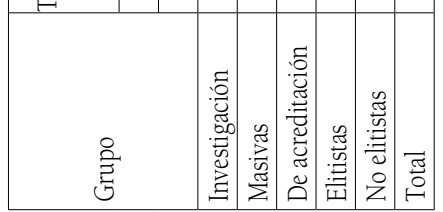


1. Columna 1: Número de instituciones clasificadas en cada uno de los grupos. Fuente: Servicio de Información de Educación Superior (SIES), 2010.

2. Columna 2: Número promedio de sedes (ubicadas en distintas ciudades) reportados por las universidades. Fuente: Índices, 2010.

3. Columna 3: Número de programas de Nivel $5 \mathrm{~A}$ y $5 \mathrm{~B}$ de la Clasificación Internacional Normalizada de la Educación (CINE) reportado por cada institución. Fuente: Índices, 2010.

4. Columna 4 y 5: Número de programas de magíster y doctorado reportado por cada institución. No se consideran especialidades médicas y odontológicas. Fuente: Índices, 2010.

5. Columna 6: Número de años de acreditación promedio vigentes al 15 de enero de 2010 para las universidades en cada uno de los grupos. El máximo de años corresponde a siete y el mínimo a cero. Fuente: Consejo Nacional de Acreditación, 2010.

6. Columna 7: Número promedio de áreas acreditadas en cada grupo vigentes al 15 de enero de 2010. Las áreas posibles de acreditación son seis: gestión institucional, docencia conducente a título, docencia de posgrado, investigación, vinculación con el medio e infraestructura y equipamiento. Fuente: Índices, 2010

7. Columnas 8 y 9: Número de matrícula total (MT), es decir, de pre y postgrado en los niveles 5A, 5B y 6 de la CINE, de las universidades incluidas en cada grupo (columna 8) y tamaño promedio de estas según la MT promedio en cada grupo. Fuente: Servicio de Información de Educación Superior (SIES), 2010. No existe omisión de instituciones.

8. Columna 10: Número de matrícula nueva (MN) ingresada el año 2010 a las universidades de cada grupo. Es un indicador aproximado del dinamismo de la matrícula (crecimiento) de cada grupo. Fuente: Servicio de Información de Educación Superior (SIES), 2010. No existe omisión de datos.

9. Columna 11: Número de titulados de pregrado (niveles 5A y 5B) de las universidades incluidas en los diferentes grupos correspondientes al año 2010. Fuente: Servicio de Información de Educación Superior (SIES), 2010.

10. Columna 12: Número de proyectos Fondecyt, concurso regular, activos en las bases de cada grupo durante el año 2009. Algunas 
instituciones incluyen información años 2008, 2007 y 2006 inclusive, por no encontrarse información actualizada. Fuente: Índices, 2010.

11. Columna 13: Número de publicaciones científicas y técnicas registradas en las bases Thompson-ISI para los años 20032008 (suma total) según se halla reportado en el Ranking Iberoamericano de Instituciones de Investigación. Fuente: Universia, 2012. Disponible en http://investigacion.universia.net/ isi/isi.html (visitada 10 de febrero del 2012).

12. Columna 14: Selectividad académica promedio de cada grupo calculada como el promedio de la selectividad académica de las universidades comprendidas en cada uno de ellos. A su turno, esta última se calcula como el porcentaje de alumnos incluidos dentro de los alrededor de 27 mil mejores puntajes de la PSU para efectos de la asignación del aporte fiscal indirecto (AFI) que ingresan al primer año en las universidades de cada uno de los grupos. Fuente: Servicio de Información de Educación Superior (SIES), 2010.

13. Columna 15, 16 y 17: Porcentaje de alumnos en la matrícula nueva del año 2010 de las correspondientes universidades que proviene directamente, vía PSU, de colegios municipales, particulares subvencionados o particulares pagados. No se incluye a los alumnos provenientes de colegios extranjeros, de otras carreras dentro de la misma institución, de otras instituciones o ingresados por otras vías especiales. Fuente: Servicio de Información de Educación Superior (SIES), 2010.

Recibido: 26/10/2012

Aceptado: 8/05/2013 